\title{
The Legendre Polynomials Associated with Bernoulli, Euler, Hermite and Bernstein Polynomials
}

\author{
Serkan Araci ${ }^{1, *}$, Mehmet Acikgoz ${ }^{2}$, Armen Bagdasaryan ${ }^{3}$, Erdoğan Şen $^{4}$ \\ ${ }^{1}$ Atatürk Street, 31290 Hatay, Turkey \\ ${ }^{2}$ University of Gaziantep, Faculty of Science and Arts, Department of Mathematics, Gaziantep, Turkey \\ ${ }^{3}$ Russian Academy of Sciences, Institute for Control Sciences, Profsoyuznaya, Moscow, Russia \\ ${ }^{4}$ Department of Mathematics, Faculty of Science and Letters, Namik Kemal University, Tekirdağ, Turkey \\ *Corresponding author: mtsrkn@hotmail.com \\ Received August 01, 2013; Revised September 10, 2013; Accepted September 18, 2013
}

\begin{abstract}
In the present paper, we deal mainly with arithmetic properties of Legendre polynomials by using their orthogonality property. We show that Legendre polynomials are proportional with Bernoulli, Euler, Hermite and Bernstein polynomials.
\end{abstract}

Keywords: Legendre polynomials, Bernoulli polynomials, Euler polynomials, Hermite polynomials, Bernstein polynomials, orthogonality

Cite This Article: Serkan Araci, Mehmet Acikgoz, Armen Bagdasaryan, and Erdoğan Şen, "The Legendre Polynomials Associated with Bernoulli, Euler, Hermite and Bernstein Polynomials." Turkish Journal of Analysis and Number Theory 1, no. 1 (2013): 1-3. doi: 10.12691/tjant-1-1-1.

\section{Introduction}

Legendre polynomials, which are special cases of Legendre functions, are introduced in 1784 by the French mathematician A. M. Legendre (1752-1833). Legendre functions are a vital and important in problems including spherical coordinates. Due to their orthogonality properties they are also useful in numerical analysis (see [9]). Besides, the Legendre polynomials, $P_{n}(x)$, are described via the following generating function:

$$
\frac{1}{\sqrt{1-2 x t+t^{2}}}=\sum_{n=0}^{\infty} P_{n}(x) t^{n}
$$

Legendre polynomials are the everywhere regular solutions of Legendre's differential equation that we can write as follows:

$$
\begin{aligned}
& \left(1-x^{2}\right) \frac{d}{d x} P_{n}(x)-2 x \frac{d}{d x} P_{n}(x)+m P_{n}(x) \\
& =\frac{d}{d x}\left[\left(1-x^{2}\right) \frac{d}{d x} P_{n}(x)\right]+m P_{n}(x)=0,
\end{aligned}
$$

where $m=n(n+1)$ and $n=0,1,2, \ldots$ Taking $x=1$ in (1) and by using geometric series, we see that $P_{n}(1)=1$, so that the Legendre polynomials are normalized.

Legendre polynomials can be generated using Rodrigue's formula as follows:

$$
P_{n}(x)=\frac{1}{n ! 2^{n}} \frac{d^{n}}{d x^{n}}\left(x^{2}-1\right)^{n} .
$$

Note that the right hand side of (2) is a polynomial (see $[3,9])$.

The Bernoulli polynomials are defined by means of the following generating function:

$$
\sum_{n=0}^{\infty} B_{n}(x) \frac{t^{n}}{n !}=\frac{t}{e^{t}-1} e^{x t},|t|<2 \pi(\text { see }[4,7]) .
$$

By (3), we know that $\frac{d B_{n}(x)}{d x}=n B_{n-1}(x)$. Taking $x=0$ in (3), we have $B_{n}(0):=B_{n}$ that stands for $n-t h$ Bernoulli number.

The Euler polynomials are known to be defined as:

$$
\sum_{n=0}^{\infty} E_{n}(x) \frac{t^{n}}{n !}=\frac{2}{e^{t}+1} e^{x t}
$$

The Euler polynomials can also be expressed by explicit formulas, e.g.

$$
E_{n}(x)=\sum_{k=0}^{n}\left(\begin{array}{l}
n \\
k
\end{array}\right) \frac{E_{k}}{2^{k}}\left(x-\frac{1}{2}\right)^{n-k},
$$

where $E_{k}$ means the Euler numbers. These numbers are expressed with the Euler polynomials through $E_{k}=2^{k} E_{k}(1 / 2)$.

Now also, we give the definition of Hermite polynomials as follows:

$$
e^{2 x t-t^{2}}=\sum_{n=0}^{\infty} H_{n}(x) \frac{t^{n}}{n !} .
$$


Let $C([0,1])$ be the space of continuous functions on $[0,1]$. For $f \in C([0,1])$, Bernstein operator for $f$ is defined by

$$
\begin{aligned}
& \mathcal{B}_{n}(f, x)=\sum_{k=0}^{n} f\left(\frac{k}{n}\right) B_{k, n}(x) \\
= & \sum_{k=0}^{n} f\left(\frac{k}{n}\right)\left(\begin{array}{l}
n \\
k
\end{array}\right) x^{k}(1-x)^{n-k},
\end{aligned}
$$

where $n, k \in \mathbb{N}^{*}:=\mathbb{N} \cup\{0\}$ and $\mathbb{N}$ is the set of natural numbers. Here $B_{k, n}(x)$ is called Bernstein polynomials, which are defined by

$$
B_{k, n}(x)=\left(\begin{array}{l}
n \\
k
\end{array}\right) x^{k}(1-x)^{n-k}, x \in[0,1] \text { (cf. [1,6].) }
$$

In [9], [3], the orthogonality of Legendre polynomials is known as

$$
\int_{-1}^{1} P_{m}(x) P_{n}(x) d x=\frac{2}{2 n+1} \delta_{m, n},
$$

where $\delta_{m, n}$ is Kronecker's delta.

In [7], by using orthogonality property of Legendre, Kim et al. effected interesting identities for them. We also obtain some interesting properties of the Legendre polynomials arising from Bernoulli, Euler, Hermite and Bernstein polynomials.

\section{Identities on the Legendre Polynomials} Arising from Bernoulli, Euler, Hermite and Bernstein Polynomials

Let $\mathcal{P}_{n}=\{q(x) \in \mathbb{Q}[x] \mid \operatorname{deg} p(x) \leq n\}$. Then we define an inner product on $\mathcal{P}_{n}$ as follows:

$$
\begin{gathered}
\left\langle q_{1}(x), q_{2}(x)\right\rangle=\int_{-1}^{1} q_{1}(x) q_{2}(x) d x, \\
\left(q_{1}(x), q_{2}(x) \in \mathcal{P}_{n}\right) .
\end{gathered}
$$

Note that $P_{0}(x), P_{1}(x), \ldots, P_{n}(x)$ are the orthogonal basis for $\mathcal{P}_{n}$. Let us now consider $q(x) \in \mathcal{P}_{n}$; then we see that

$$
q(x)=\sum_{k=0}^{n} C_{k} P_{k}(x),
$$

where the coefficients $C_{k}$ are defined over the field of real numbers.

From the above, we readily see that

$$
\begin{aligned}
C_{k} & =\frac{2 k+1}{2}\left\langle q(x), P_{k}(x)\right\rangle \\
& =\frac{2 k+1}{2} \int_{-1}^{1} P_{k}(x) q(x) d x \\
& =\frac{2 k+1}{k ! 2^{k+1}} \int_{-1}^{1} \frac{d^{k}}{d x^{k}}\left(x^{2}-1\right)^{k} q(x) d x .
\end{aligned}
$$

By (9) and (10), we have the following proposition.

Proposition 2.1. Let $q(x) \in \mathcal{P}_{n} \quad$ and $q(x)=\sum_{k=0}^{n} C_{k} P_{k}(x)$, then

$$
\begin{aligned}
& C_{k}=\frac{2 k+1}{k ! 2^{k+1}} \int_{-1}^{1}\left(\frac{d^{k}}{d x^{k}}\left(x^{2}-1\right)^{k}\right) q(x) d x \text { (see [7]). } \\
& \text { If we take } q(x)=x^{n} \text { in Proposition (2.1), the }
\end{aligned}
$$
coefficients $C_{k}$ can be found as

$$
C_{k}=\frac{(2 k+1) 2^{k+1}}{(n+k+2) !} \frac{n !\left(\frac{n+k+2}{2}\right) !}{\left(\frac{n-k}{2}\right) !} \quad \text { (see [7]). }
$$$$
\text { for } n-k \equiv 0(\bmod 2)
$$

Let $q(x)=B_{n}(x)$. Then by using Proposition 2.1 and (11), we have

$$
C_{k}=\frac{2 k+1}{k ! 2^{k+1}} \int_{-1}^{1}\left(\frac{d^{k}}{d x^{k}}\left(x^{2}-1\right)^{k}\right) B_{n}(x) d x
$$

where $B_{n}(x)$ are the aforementioned Bernoulli polynomials that can be expressed through Bernoulli numbers $B_{n}$ as follows:

$$
B_{n}(x)=\sum_{j=0}^{n}\left(\begin{array}{l}
n \\
j
\end{array}\right) B_{n-j} x^{j}
$$

From this, we have

$$
\begin{aligned}
& C_{k}=\sum_{j=0}^{n}\left(\begin{array}{c}
n \\
j
\end{array}\right) B_{n-j}\left[\int_{-1}^{1}\left(\frac{d^{k}}{d x^{k}}\left(x^{2}-1\right)^{k}\right) x^{j} d x\right] \\
& =\left(2^{k+2} k+2^{k+1}\right) \sum_{j=0}^{n} \frac{j !\left(\begin{array}{c}
n \\
j
\end{array}\right)\left(\frac{j+k+2}{2}\right) !}{\left(\frac{j-k}{2}\right) !(j+k+2) !} B_{n-j} \\
& \text { for } j-k \equiv 0(\bmod 2) .
\end{aligned}
$$

Therefore we have the following theorem.

Theorem 2.2. Let $B_{n}(x)=\sum_{k=0}^{n} C_{k} P_{k}(x) \in \mathcal{P}_{n}$. Then we have

$$
\begin{aligned}
& B_{n}(x) \\
&=2 \sum_{k=0}^{n}\left(\sum_{j-k \equiv 0(\bmod 2)}^{n} \frac{j !\left(\begin{array}{c}
n \\
j
\end{array}\right)\left(\frac{j+k+2}{2}\right) !}{\left(\frac{j-k}{2}\right) !(j+k+2) !} B_{n-j}\right)
\end{aligned} P_{k}(x) .
$$

Let $H_{n}(x) \in \mathcal{P}_{n}$. By Proposition 2.1 and (11), we have the following theorem.

Theorem 2.3. Let $H_{n}(x)=\sum_{k=0}^{n} C_{k} P_{k}(x) \in \mathcal{P}_{n}$. Then we have 


$$
\begin{aligned}
& H_{n}(x) \\
& =\sum_{k=0}^{n}\left(\begin{array}{l}
\left(2^{k+2} k+2^{k+1}\right) \\
\sum_{j-k \equiv 0(\bmod 2)}^{n} \frac{2^{j}\left(\begin{array}{c}
n \\
j
\end{array}\right) j !\left(\frac{j+k+2}{2}\right) !}{(j+k+2) !\left(\frac{j-k}{2}\right) !} H_{n-j}
\end{array}\right) P_{k}(x) .
\end{aligned}
$$

Let the Bernstein polynomials $B_{j, n}(x) \in \mathcal{P}_{n}$. By Proposition 2.1 and (11), we have following theorem.

Theorem 2.4. Let $B_{j, n}(x)=\sum_{k=0}^{n} C_{k} P_{k}(x) \in \mathcal{P}_{n}$. We have

$$
\begin{aligned}
& B_{j, n}(x) \\
& =\sum_{k=0}^{n}\left(\begin{array}{c}
\left(2^{k+2} k+2^{k+1}\right) \\
\sum_{l+j-k \equiv 0(\bmod 2)}^{n-j} \frac{\left(\begin{array}{c}
n-j \\
l
\end{array}\right)(-1)^{l}(l+j) !\left(\frac{l+j+k+2}{2}\right)}{(l+j+k+2) !\left(\frac{l+j-k}{2}\right) !}
\end{array}\right) P_{k}(x) .
\end{aligned}
$$

The following equality is defined by Kim et al. in [7]:

$$
\begin{aligned}
& \sum_{k=0}^{n} B_{k}(x) B_{n-k}(x) \\
= & \frac{2}{n+2} \sum_{l=0}^{n-2}\left(\begin{array}{c}
n+2 \\
l
\end{array}\right) B_{n-l} B_{l}(x)+(n+1) B_{n}(x) .
\end{aligned}
$$

Let $\sum_{k=0}^{n} B_{k}(x) B_{n-k}(x) \in \mathcal{P}_{n}$. By Proposition 2.1 and (11), we get the following theorem.

Theorem 2.5. Let $\sum_{k=0}^{n} B_{k}(x) B_{n-k}(x) \in \mathcal{P}_{n}$. Then we have

$$
\begin{aligned}
& \sum_{k=0}^{n} B_{k}(x) B_{n-k}(x) \\
= & \sum_{k=0}^{n}\left(2^{k+2} k+2^{k+1}\right)\left\{\frac{2}{n+2} \sum_{l=0}^{n-2} \sum_{j-k \equiv 0(\bmod 2)}^{l} B_{n-l} B_{n-j}\right. \\
& \times \frac{\left(\begin{array}{c}
n+2 \\
l
\end{array}\right)\left(\begin{array}{l}
l \\
j
\end{array}\right)(j) !\left(\frac{j+k+2}{2}\right) !}{\left(\frac{j-k}{2}\right) !(j+k+2) !} \\
+ & \left.(n+1) \quad \sum_{l-k \equiv 0(\bmod 2)}^{l}\left(\begin{array}{l}
n \\
l
\end{array}\right) B_{n-l} \frac{l !\left(\frac{l+k+2}{2}\right) !}{\left(\frac{l-k}{2}\right) !(l+k+2) !}\right\} P_{k}(x) .
\end{aligned}
$$

Let $q(x)=\sum_{k=0}^{n} E_{k}(x) E_{n-k}(x) \in \mathcal{P}_{n}$. In [8], Kim et al. derived convolution formula for the Euler polynomials as

$$
\begin{aligned}
& \sum_{k=0}^{n} E_{k}(x) E_{n-k}(x) \\
= & -\frac{4}{n+2} \sum_{l=0}^{n}\left(\begin{array}{c}
n+2 \\
l
\end{array}\right) E_{n-l+1}(0) B_{l}(x) .
\end{aligned}
$$

By Proposition 2.1 and (11), we get the following theorem.

Theorem 2.6. The following equality holds true:

$$
\begin{aligned}
& \sum_{k=0}^{n} E_{k}(x) E_{n-k}(x) \\
& =-\frac{8}{n+2} \sum_{k=0}^{n}\left(2^{k+1} k+2^{k}\right)
\end{aligned}
$$

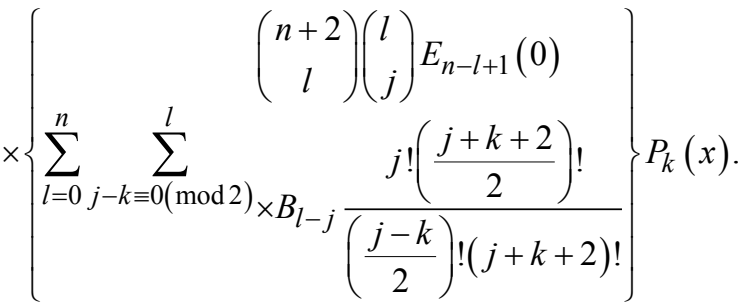

Remark 2.7. By using Theorem 2.1, we can find many interesting identities for the special polynomials in connection with Legendre polynomials.

\section{References}

[1] S. Araci, D. Erdal and J. J. Seo, A study on the fermionic p-adic qintegral representation on $\mathbb{Z}_{\mathrm{P}}$ associated with weighted q-Bernstein and q-Genocchi polynomials, Abstract and Applied Analysis, Volume 2011 (2011), Article ID 649248, 10 pages.

[2] A. Bagdasaryan, An elementary and real approach to values of the Riemann zeta function, Phys. Atom. Nucl. 73, 251-254, (2010).

[3] W. N. Bailey, On the product of two Legendre polynomials, Proc. Cambridge Philos. Soc. 29 (1933), 173-177.

[4] B. C. Kellner, On irregular prime power divisors of the Bernoulli numbers, Mathematics of Computation, Volume 76, Number 257, January 2007, Pages 405-441.

[5] T. Kim, Some identities on the q-Euler polynomials of higher order and q-stirling numbers by the fermionic p-adic integral on $\mathbb{Z}$ p, Russian J. Math. Phys. 16 (2009), 484-491.

[6] T. Kim, J. Choi, Y. H. Kim and C. S. Ryoo, On q-Bernstein and qHermite polynomials, Proc. Jangjeon Math. Soc. 14 (2011), no. 2, 215-221.

[7] D. S. Kim, S.-H. Rim and T. Kim, Some identities on Bernoulli and Euler polynomials arising from orthogonality of Legendre polynomials, Journal of Inequalities and Applications 2012, 2012:227

[8] D. S. Kim, T. Kim, S.-H. Lee, Y.-H. Kim, Some identities for the product of two Bernoulli and Euler polynomials. Adv. Diff. Equ. 2012; 2012:95.

[9] L. C. Andrews, Special Functions of Mathematics for Engineerings, SPIE Press, 1992, pages 479. 